\title{
Oncomedicine
}

2016; 1: 18-24. doi: 10.7150/oncm.16928

Review

\section{Head and Neck Cancer Therapy from Bench to Bedside}

\author{
Ilias Karapantzos ${ }^{1}$, Paul Zarogoulidis ${ }^{\circledR}{ }^{\bowtie}$, Michail Karanikas³, Vasilis Thomaidis ${ }^{4}$, Charalampos \\ Charalampidis ${ }^{5}$, Chrysa Karapantzou ${ }^{1}$ \\ 1. Ear, Nose and Throat Department, "Saint Luke" Private Hospital, Panorama, Thessaloniki, Greece \\ 2. Pulmonary Department-Oncology Unit, “G. Papanikolaou” General Hospital, Aristotle University of Thessaloniki, Thessaloniki, Greece \\ 3. General Surgery Department, "Genisis" Private Clinic, Thessaloniki, Greece \\ 4. Department of Maxillofacial Surgery, University General Hospital of Alexandroupolis, Alexandroupolis, Greece \\ 5. Department of Anatomy, Democritus University of Thrace, Alexandroupolis, Greece.
}

$\square$ Corresponding author: Paul Zarogoulidis, M.D, Ph. D, Pulmonary Department-Oncology Unit, “G. Papanikolaou” General Hospital, Aristotle University of Thessaloniki, Thessaloniki, Greece. Fax: 00302310992424; Mobile: 00306977271974; E-mail: pzarog@hotmail.com

(C) Ivyspring International Publisher. This is an open access article distributed under the terms of the Creative Commons Attribution (CC BY-NC) license (https://creativecommons.org/licenses/by-nc/4.0/). See http://ivyspring.com/terms for full terms and conditions.

Received: 2016.07.21; Accepted: 2016.08.23; Published: 2016.09.05

\begin{abstract}
Nowadays treatment of laryngeal and hypopharyngel cancer is not defined only by surgical resection. Multimodality treatment approach is considered the best approach for patients. Currently treatment includes chemotherapy, radiotherapy, and surgery. The goal with combination treatment approach is to preserve organ and function. In the past years unfortunately we had to deal with mutilating and function destroying treatment. In the past 25 years, evidences from large randomized trials presented data that organ preservation studies using sequential and concomitant radio-chemotherapy do not compromise survival when compared with surgery followed by radiotherapy. The side effects from multimodality treatment approach has to take into account and the final goal has to be not only organ preservation but also function preservation. In the current review we focus on the most common treatment options. We conclude that there is an urgent need to refine the definition of a functional organ and to refine recommendations for evaluating treatment response.
\end{abstract}

Key words: Head and neck cancer, Concurrent radio-chemotherapy, Induction chemotherapy, Organ preservation surgery, Molecular therapy

\section{Introduction}

It is known that head and neck cancer is the sixth most common cancer worldwide. The incidence has been recorded to be over 500,000 new cases each year.(1) Usually up to $90 \%$ of the histology type is squamous cell carcinoma originating from the mucosal epithelia. It has been observed that patients with squamous cell carcinoma (HNSCC) diagnosed at early stages (stage I/II) can achieve long-term survival benefit from single modality treatment either surgery or radiotherapy (RT). On the other hand, patients with locally advanced HNSCC (stage III/IV) a multi-modality treatment approach is necessary including surgical resection, chemotherapy, and radiotherapy are generally required.

In locally advanced stage of HNSCC surgery is followed by combined chemo-radiotherapy and remains the main treatment of choice for these patients. Chemotherapy (CT) has increased locoregional control and reduces the incidence of distant metastasis. Chemotherapy also has increased effect in the case where the tumor has an adequate blood supply; however, it is less effective as the tumor vascularity has been compromised by previous surgery or radiotherapy. $(2,3)$ Induction chemotherapy (IC) is considered an effective way for down staging the disease in case of advanced or aggressive 
malignancies, but also improves the chance of local tumor eradication by either surgical extirpation combined with postoperative radiation \pm concomitant chemotherapy (CCRT) or definitive RT/CCRT. The benefit of induction chemotherapy is clear in some malignancies; however, the clinical value of induction chemotherapy in HNSCC still remains unclear. To date the presented data do not show a clear benefit of induction chemotherapy in HNSCC. $(4,5)$ During the last ten years several randomized control trials have reported a benefit of adding induction chemotherapy to standard therapy regimens for HNSCC. Current multimodality treatment approaches will be presented in the current work.

\section{Concurrent chemo-radiotherapy versus induction chemotherapy}

It has been reported in the Radiation Therapy Oncology Group (RTOG) 91-11 study $(6,7)$ that concurrent chemotherapy and radiotherapy (RT) multimodality treatment was superior to sequential therapy or RT alone for stage III or stage IV laryngeal cancers. 547 patients with T2, T3, or "low-volume" T4 tumors were randomly assigned to one of the three study groups. During a 2 assessment it was observed that $(88 \%)$ of the patients who had radiotherapy with concurrent chemotherapy had an intact larynx and the proportion higher than the proportions in the groups receiving sequential therapy $(75 \%, P=0.005)$ or radiotherapy alone $(70 \%, P<0.001)$. It was also observed that the rate of loco-regional control was higher with radiotherapy and concurrent cisplatin. The 5-year results (7) indicate that there is an improvement in laryngectomy-free-survival for both sequential therapy and radiotherapy with concurrent chemotherapy treatments when compared to radiotherapy alone. Regarding the endpoint of laryngeal preservation and loco-regional control, radiotherapy with concurrent chemotherapy still remains superior with no advantage seen over the addition of induction chemotherapy to radiation alone. However, there is no improvement on the rate of distant metastases. Moreover, it has to be stated that the morbidity of nonsurgical treatments of laryngeal cancer was significant in this trial, and associated with a $3 \%$ risk of treatment-related death. Until now it is proposed that radiotherapy with concurrent chemotherapy should be considered as the standard of care for patients desiring laryngeal preservation and that laryngectomy should be performed only as a salvage therapy. However, acute toxicity was observed to be greater than threefold increase after the addition of chemotherapy to radiation without an improvement in survival.(8) The
EORTC 24954 trial compared alternating chemo-radiotherapy and induction chemotherapy but there was no significant difference between both arms regarding survival and larynx preservation.(9) It was observed that multimodality non-operative treatment such as chemotherapy or radiotherapy are successful but severe late toxicity is common.

The toxicity in 230 patients was $43 \%$. However, concomitant use of chemotherapy and radiation has presented favorable results for loco-regional control and survival benefits, when compared to RT alone. Favorable results are observed when a high-dose single-agent cisplatin and concurrent radiotherapy are administered to patients with advanced nasopharynx cancer, un-resectable head and neck tumors, and as a postoperative adjuvant for patients with high-risk of recurrence.(10,11) Previously Concomitant chemo-radiotherapy was administered with taxane. A phase II multicenter study reported high organ-preservation rate with taxane-based chemotherapy for oropharynx but the case was not the same for larynx cancer patients.(12) The toxicity rate observed for these patients was low. Furthermore, a Spanish group conducted a phase II trial of concomitant chemo-radiotherapy with weekly docetaxel for advanced head and neck head and neck cancer (13), however; although the results regarding effectiveness were the same, toxicity was higher when compared to exclusive cisplatin schemes. Neoadjuvant chemotherapy followed by concomitant chemo-radiotherapy has been investigated with the aim to increase survival and laryngeal preservation. First studies used cisplatin and 5-fluoruracil as the main components of induction chemotherapy and usually consisted of three cycles. After the induction chemotherapy patients with residual disease received salvage surgery. The "responders" proceeded to concomitant chemo-radiotherapy. In the study by Mantz et al. (14) added leucovorin and interferon-alpha $2 b$ to the induction regimen. The concomitant chemo-radiotherapy consisted of seven or eight cycles of 5-Fluouracil, hydroxyurea, and a total RT dose of $70 \mathrm{~Gy}$. Thirty-two laryngeal cancer patients with predominantly stage IV disease were included and complete remission was reported in $59 \%$. The 5-year old overall survival (OS) was $47 \%$ and loco-regional control was achieved in $78 \%$ of patients. Voice preservation with disease control was observed to be $75 \%$ at 5 years. Only two patients had to undergo laryngectomy during treatment and follow-up and no distant metastases were observed. Unfortunately, treatment-related toxicity was observed in two patients. In this study it was concluded that the treatment resulted in high rates of 
disease cure and voice preservation in a group of patients that usually performed poorly in both clinical and functional outcomes.

The GORTEC 2000-01 trial (15) compared a more intensive induction chemotherapy regimen where docetaxel was added to the conventional cisplatin/5-fluoruracil regimen. The median follow-up was 36 months and the 3-year larynx preservation rate was around $70 \%$ which was significantly higher with the triplet induction chemotherapy (Taxane Platinum Fluoruracil) than with the doublet (Platinum Fluoruracil). Patients in the (Taxane Platinum Fluoruracil) group had more severe neutropenia, whereas patients in the PF group had more thrombocytopenia, stomatitis, and creatinine elevation. The overall response was $80.0 \%$ in the (Taxane Platinum Fluoruracil) group versus $59.2 \%$ in the (Taxane Platinum Fluoruracil) group. It was observed in this study that patients with advanced larynx and hypopharynx carcinomas, Taxane Platinum Fluoruracil induction chemotherapy was superior to the Platinum Fluoruracil regimen for overall response rate. The results presented in this study suggest that larynx preservation could be achieved for a higher proportion of patients. However, no significant difference was observed for the survival rate. In the study by Posner et al. (16) the data presented showed that locally advanced laryngeal and hypopharyngeal cancer with sequential therapy after induction Taxane Platinum Fluoruracil significantly improved survival and progression-free survival versus Taxane Platinum Fluoruracil. It was also observed that operable patients, Taxane Platinum Fluoruracil also significantly improved laryngectomy free survival and progression-free survival. The authors of this study proposed the use of sequential Taxane Platinum Fluoruracil TPF followed by carboplatin chemo-radiotherapy as a treatment option for organ preservation or additionally improve survival in locally advanced laryngeal and hypopharyngeal cancer. In a recently presented study TAX324 an association between HPV and OS in oropharyngeal cancer was examined (5-year follow-up).(17, 18) It was observed that the survival benefit of sequential therapy with Taxane Platinum Fluoruracil remains significantly superior to Platinum Fluoruracil beyond 5 years of continuing follow up. It was also observed that patients over age 55 tend to benefit more from treatment with Taxane Platinum Fluoruracil than younger patients and the benefit with Taxane Platinum Fluoruracil becomes more clear with a longer follow up in patients with oropharynx tumors. It is known that HPV positive oropharyngeal cancer has a different demography, biology, and OS compared to HPV negative oropharyngeal cancer. It has been observed that HPV positive group has an excellent long term survival and predicts durable long-term OS. These data support the development of different therapeutic approaches for HPV positive and HPV negative oropharyngeal cancer. The European TAX 323 study group (EORTC 24971) (19) also compared Taxane Platinum Fluoruracil with Platinum Fluoruracil as induction chemotherapy in patients with loco-regionally advanced and/or un-resectable disease. Treatment with Taxane Platinum Fluoruracil resulted in a reduction in the risk of death of $27 \%(P=0.02)$, with a median OS of 18.8 months, as compared with 14.5 months in the Platinum Fluoruracil group. Data presented that the addition of docetaxel to the standard regimen of cisplatin and fluorouracil, improved the progression-free survival and OS in these patients. In the case treatment failure for nonsurgical treatments, then surgical total laryngectomy will be indicated. It has been observed that salvage laryngectomy is associated with an increased risk of wound complications compared to those performed before radiation. Recent data report up to in $27 \%$ of major wounds in patients, with a range between 5 and $48 \%$ in a recent review of the literature.(20) Pharyngocutaneous fistulas have been reported in $15-80 \%$ of patients undergoing salvage laryngectomy. A fatal and catastrophic complication arises if a fistula results in rupture of the carotid artery. This is complication is rare and in the absence of antecedent radiation should be taken into consideration carefully.

\section{Molecular therapy}

Epidermal Growth Factor Rreceptor (EGFR) inhibition is one of the new strategies focusing on molecular targets. EGFR and its ligands have been recognized as critical proteins in the development and survival of epithelial tissue. It has been observed that squamous cell carcinomas of the head and neck tend to express high levels of EGFR. Inhibition of EGFR signaling by small molecules, monoclonal antibodies or antisense oligonucleotides has demonstrated anticancer effectiveness.(21) On the other hand cetuximab, a monoclonal antibody against EGFR, and small-molecule tyrosine kinase inhibitor, such as gefitinib or erlotinib, have to be proven effective in clinical applications. A recent publication of a randomized controlled trial of radiotherapy with and without concomitant cetuximab showing significantly improved OS ( 55 vs. $45 \%$ at 3 years, $P=0.03$ ). This study led to the FDA approval of cetuximab in combination with radiotherapy for the primary treatment of head and neck squamous cell 
carcinoma.(22) In this study the median duration of loco-regional control was 24.4 months among patients treated with cetuximab plus radiotherapy and 14.9 months among those given radiotherapy alone. These results presented promising results in patients with oropharyngeal primary tumors, however; little difference was observed for patients with laryngeal or hypopharyngeal cancers. Currently a number of clinical trials are in progress evaluating combinations of cetuximab and cisplatin-based chemotherapy as induction or concomitant chemo-radiation.

\section{Organ preservation surgery}

Currently primary laryngectomy remains the best treatment option for a number of patients with advanced laryngeal cancer. This is because those with cartilaginous destruction and organ dysfunction are poor candidates for chemo-radiation. Patients who undergo chemo-radiation must be capable of compliance and must have good performance status. Technological advances provide improved organ preservation strategies in surgery. Surgeons currently are able to maintain physiological functions of larynx such as respiration, speech and swallowing without compromising the locoregional control.(23) Laryngeal preservation rates of $90 \%$ and local control rates of 91\% for 118 T3N0-3 laryngeal cancers are reported.(24) Minimum requirements for a surgeon who performs organ preservation surgery are: (1) thoroughly assessing the cancer clinically and radiologically; (2) comprehension of static and dynamic anatomy of the laryx; and (3) training to competently perform the chosen surgical technique. In the study by Tufano et. al. (25) key principles are reported as follows:

1. Ability to confidently predict the extent of the tumor including dynamic interpretation of laryngeal function.

2. Local control: Early detection of residual or recurrent disease. Regular and close follow up of the patient is very important.

3. The cricoarytenoid unit is the basic functional unit of the larynx. Preservation of the cricoarytenoid unit is a potential candidate for organ preservation laryngeal surgery.

4. Necessity of resection of normal tissue in organ preservation surgery to achieve consistent functional outcomes in terms of speech and swallowing. Currently different surgical techniques are performed for different tumor extensions. The vertical partial laryngectomy (VPL) is usually used in treatment of early glottis cancer.(26) The usual contraindications are the involvement of the crico-arytenoid joint or the thyroid cartilage and of more than $1 / 3$ rd of the contralateral vocal cord.(27) The supraglottic laryngectomy (SGL), was first described by Alonso et. al. and is a procedure for intermediate stage supraglottic cancer.(28) After surgery the voice quality is normally good as the vocal cords can be preserved. The supracricoid laryngectomy (SCPL) bridges the gap between partial open procedures and total laryngectomy.(25) It has been observed that when comparing VPL to SCPL a more comprehensive resection of the para-glottic space is available. SCPL is used in glottic, transglottic or supraglottic cancer up to T3 if SGL is inappropriate. Currently this technique is used in selected $\mathrm{T} 4$ larynx-limited invasion with mini endoscopic surgery as management. This management plays an evolving role in laryngeal cancer treatment. In the recent years. There was an evolution of minimally invasive surgery for tumors of the upper aerodigestive tract with the development of the $\mathrm{CO} 2$ laser coupled to an operative microscope initially.(29) In several studies the effectiveness of this endoscopic treatment was evaluated (CO2 laser) for the resection of oropharyngeal, hypopharyngeal and laryngeal cancers.(29-31) The reports about achieving local control rate are very encouraging in the range of $80-94 \%$ and organ preservation rate has been observed up to $94 \%$ of cases in endoscopic laser microsurgery.(32) In recent studies the transoral approach which is supported by the daVinci Surgical System has been adapted for use in head and neck cancers.(33) The important reason for endoscopic techniques versus open techniques is that the endoscopic techniques preserve the sensory innervation and normal suspension of larynx. In general, the recovery from minimally invasive surgery is rapid. Patients usually have shorter hospitalization and better swallowing outcome. Moreover, a favorable impact on their psychological profile has been observed.

\section{Discussion}

Clinical research has shifted treatment options in the treatment of larynx and hypopharynx cancer. The current trend based on recent data is to perform less surgeries.(34-36) However, there is no randomized trial of organ preservation in advanced laryngeal cancer that demonstrated improved survival with non-surgical treatment.(6) Novel multimodality treatments are offered as an "alternative" treatment approach in order to improve functional and quality of life for patients in the form of laryngeal preservation. However, still the discussion about larynx preservation remains controversial. In any case the benefits should be well balanced with cancer 
control and possible adverse events.

In the study by Hoffman et. al. (37) changes in the patterns of care, and survival in laryngeal cancer were presented for the United States. Data presented indicated that the use of chemo-radiotherapy on 158,426 larynx cancers had increased and the use of surgery was decreased from mid 1980s to mid 1990s. However, survival progressively decreased over this period. Almost the same conclusion was observed for stage IV larynx cancer in the study by Chen et al. (38) In this study patients with stage IV disease, and total laryngectomy had increased survival compared with chemo-radiotherapy or radiotherapy alone. Moreover, it was observed that for stage III patients both total laryngectomy and chemo-radiotherapy overall survival was improved. In the study by Hoffman et al. (37) the differences between surgery and radiation versus chemo-radiation were much

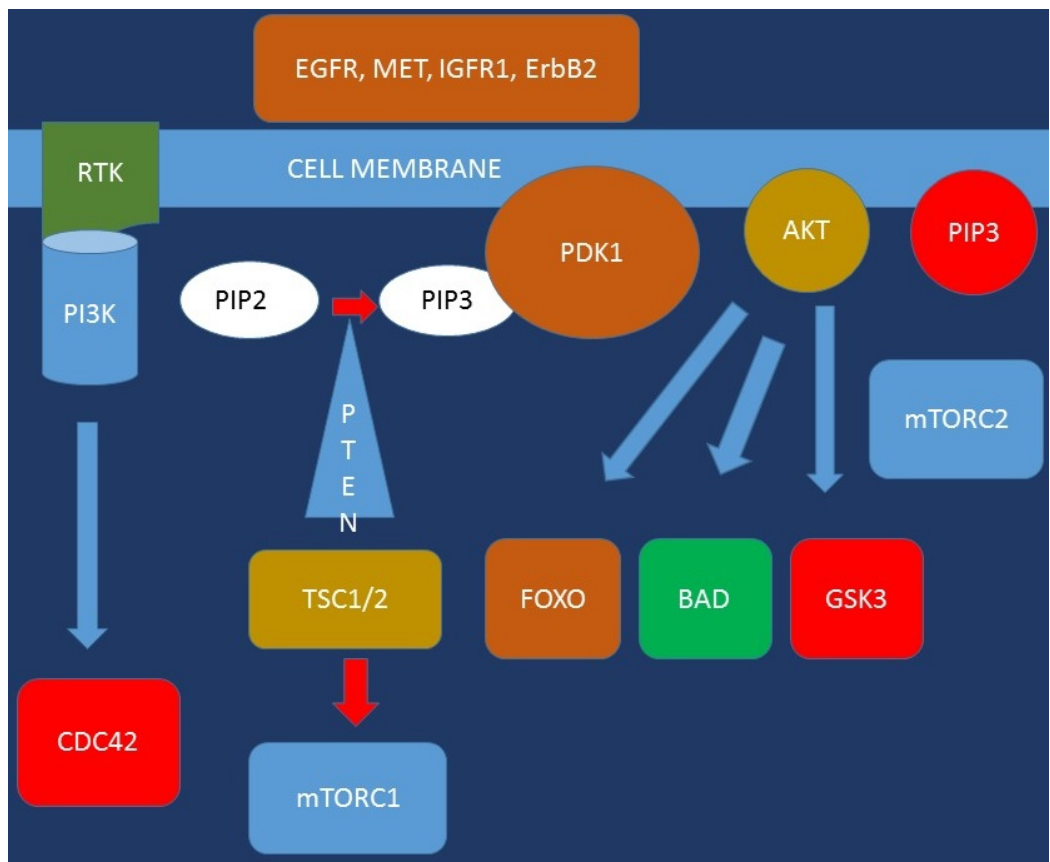

Figure 1. Growth signals of future targeted therapies under consideration.

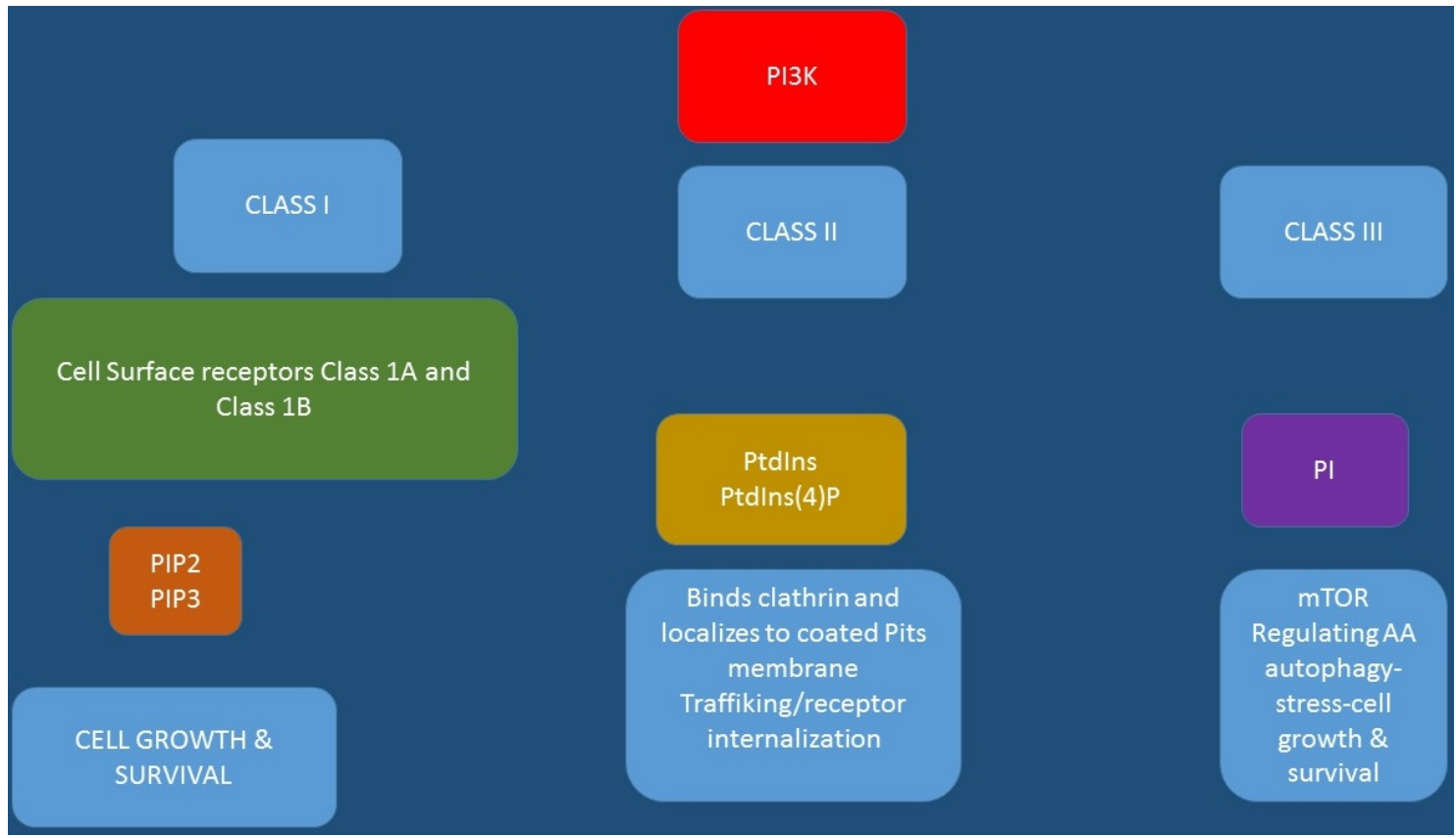

Figure 2. Targeted treatment mode of binding. 
different. A 5\% overall difference was observed in favor of surgery and radiation for the entire T3 group. However, only a significant difference was found in comparing surgery with/without radiation to radiation alone $(P<0.05)$. No statistically significant difference was observed between surgery alone or surgery plus radiation compared with chemo-radiation $(P=0.503$ and $P=0.067$, respectively). In a large subgroup of glottis T3N0 patients identical survival rates were observed (54.7 and $65.6 \%$ ) for surgery and radiation versus chemo-radiation. Upon observation of the subgroup of patients with supraglottic T3Np and T4N0 tumors, survival rates actually had improved overall over the 1990-1996 time period. This occurred with the first increases in the use of chemo-radiation therapy were performed. Furthermore, the highest decline in the survival for supraglottic cancer patients was observed among patients in the early stage who would not have received chemo-radiation. There are enormous discrepancies between the different published trials in terms of eligibility criteria, primary endpoints selection/definition and secondary endpoints. Until now the terminology of larynx preservation is not clear. One can consider a very restrictive definition for larynx preservation is for example a larynx without tumor, tracheotomy, or use of a feeding tube.(9) There is the case where anatomic organ preservation is considered tracheotomy tube- and gastrostomy tube-dependent. There are studies which consider "larynx preservation" as "larynx in place" without taking into consideration the tracheotomy or feeding tube into account. In some studies, the function of the larynx is abandoned and several studies do not included survival in the primary endpoint.

Currently a multidisciplinary expert panel has extensively reviewed the literature and developed evidence-based clinical practice guideline and defined the organ preservation "larynx".(39) The panel indicated that the use of larynx-preservation approaches is for selected patients and should not in any way compromise survival benefit. To date, no larynx-preservation approach offers a survival advantage compared to total laryngectomy and adjuvant therapy with rehabilitation. The panel recommends for T1 or T2 laryngeal cancer, with rare exception, is an initial treatment with intent to preserve the larynx. Regarding patients with T3 or T4 disease without tumor invasion through cartilage into soft tissues, larynx-preservation approach is considered appropriate, and the standard treatment option, is concurrent chemo-radiotherapy therapy.(39) Currently the use of neo-adjuvant chemotherapy in locally advanced squamous cell cancer of the head and neck is under debate. Still there is no clear definition between induction chemotherapy and concomitant chemo-radiotherapy. Ongoing studies will elucidate whether taxane-based neoadjuvant chemotherapy is appropriate for locally advanced laryngeal cancer.(40) Currently concurrent chemo-radiation is not considered the standard of care, but is an acceptable alternative to sequential chemo-radiation or to total laryngectomy since concurrent chemo-radiation has not been directly compared to total laryngectomy. A multidisciplinary treatment approach should be proposed to the patient, the board should fully discuss with the patient the advantages and disadvantages of larynx-preservation options compared with treatments that include total laryngectomy.

Treating physicians have to decide their treatment approach on a case by case basis, since each patient has a variety of characteristics (demography, biology stage).

Currently there are novel targeted treatments that are under way and novel technological equipment used during surgery which in many cases are very expensive, cost effectiveness is an issue that has to be considered.

\section{Competing Interests}

The authors have declared that no competing interest exists.

\section{References}

1. Jemal A, Siegel R, Ward E, Hao Y, Xu J, Thun MJ. Cancer statistics, 2009. CA: a cancer journal for clinicians. 2009;59(4):225-49.

2. Holsinger FC. Swing of the pendulum: optimizing functional outcomes in larynx cancer. Current oncology reports. 2008;10(2):170-5.

3. Pignon JP, Bourhis J, Domenge C, Designe L. Chemotherapy added to locoregional treatment for head and neck squamous-cell carcinoma: three meta-analyses of updated individual data. MACH-NC Collaborative Group. Meta-Analysis of Chemotherapy on Head and Neck Cancer. Lancet. 2000;355(9208):949-55.

4. Pignon JP, le Maitre A, Bourhis J, Group M-NC. Meta-Analyses of Chemotherapy in Head and Neck Cancer (MACH-NC): an update. International journal of radiation oncology, biology, physics. 2007;69(2 Suppl):S112-4.

5. Induction chemotherapy plus radiation compared with surgery plus radiation in patients with advanced laryngeal cancer. The Department of Veterans Affairs Laryngeal Cancer Study Group. The New England journal of medicine. 1991;324(24):1685-90.

6. Forastiere AA, Goepfert H, Maor M, Pajak TF, Weber R, Morrison W, et al. Concurrent chemotherapy and radiotherapy for organ preservation in advanced laryngeal cancer. The New England journal of medicine. 2003;349(22):2091-8

7. Kelsen DP, Winter KA, Gunderson LL, Mortimer J, Estes NC, Haller DG, et al. Long-term results of RTOG trial 8911 (USA Intergroup 113): a random assignment trial comparison of chemotherapy followed by surgery compared with surgery alone for esophageal cancer. Journal of clinical oncology: official journal of the American Society of Clinical Oncology. 2007;25(24):3719-25.

8. Trotti A, Pajak TF, Gwede CK, Paulus R, Cooper J, Forastiere A, et al. TAME: development of a new method for summarising adverse events of cancer treatment by the Radiation Therapy Oncology Group. The Lancet Oncology. 2007;8(7):613-24.

9. Lefebvre JL, Rolland F, Tesselaar M, Bardet E, Leemans CR, Geoffrois L, et al. Phase 3 randomized trial on larynx preservation comparing 
sequential vs alternating chemotherapy and radiotherapy. Journal of the National Cancer Institute. 2009;101(3):142-52.

10. Adelstein DJ, Rodriguez CP. Current and emerging standards of concomitant chemoradiotherapy. Seminars in oncology. 2008;35(3):211-20.

11. Forastiere AA. Chemotherapy in the treatment of locally advanced head and neck cancer. Journal of surgical oncology. 2008;97(8):701-7.

12. Cmelak AJ, Li S, Goldwasser MA, Murphy B, Cannon M, Pinto H, et al. Phase II trial of chemoradiation for organ preservation in resectable stage III or IV squamous cell carcinomas of the larynx or oropharynx: results of Eastern Cooperative Oncology Group Study E2399. Journal of clinical oncology: official journal of the American Society of Clinical Oncology. 2007;25(25):3971-7.

13. Biete Sola A, Marruecos Querol J, Calvo Manuel FA, Verger Fransoy E, Rovirosa Casino A, Grau de Castro JJ, et al. Phase II trial: concurrent radio-chemotherapy with weekly docetaxel for advanced squamous cell carcinoma of head and neck. Clinical \& translational oncology: official publication of the Federation of Spanish Oncology Societies and of the National Cancer Institute of Mexico. 2007;9(4):244-50.

14. Mantz CA, Vokes EE, Kies MS, Mittal B, Witt ME, List MA, et al. Sequential induction chemotherapy and concomitant chemoradiotherapy in the management of locoregionally advanced laryngeal cancer. Annals of oncology: official journal of the European Society for Medical Oncology / ESMO. 2001;12(3):343-7.

15. Pointreau Y, Garaud P, Chapet S, Sire C, Tuchais C, Tortochaux J, et al. Randomized trial of induction chemotherapy with cisplatin and 5-fluorouracil with or without docetaxel for larynx preservation. Journal of the National Cancer Institute. 2009;101(7):498-506.

16. Posner MR, Norris CM, Wirth LJ, Shin DM, Cullen KJ, Winquist EW, et al. Sequential therapy for the locally advanced larynx and hypopharynx cancer subgroup in TAX 324: survival, surgery, and organ preservation. Annals of oncology: official journal of the European Society for Medical Oncology / ESMO. 2009;20(5):921-7.

17. Posner MR LJ, Goloubeva O, Tan M, Schumaker L. Human NeaOcOiT. Abstracts pHasAM(Suppl):5525.

18. Lorch JH PM, Goloubeva O, Cullen KJ, Sarlis NJ. Trial ReaL-trfTapI, advanced ostcTtPil, ASCO LsccothanH(Suppl):5512.

19. Vermorken JB, Remenar E, van Herpen C, Gorlia T, Mesia R, Degardin $\mathrm{M}$, et al. Cisplatin, fluorouracil, and docetaxel in unresectable head and neck cancer. The New England journal of medicine. 2007;357(17):1695-704.

20. Goodwin WJ, Jr. Salvage surgery for patients with recurrent squamous cell carcinoma of the upper aerodigestive tract: when do the ends justify the means? The Laryngoscope. 2000;110(3 Pt 2 Suppl 93):1-18.

21. Pomerantz RG, Grandis JR. The epidermal growth factor receptor signaling network in head and neck carcinogenesis and implications for targeted therapy. Seminars in oncology. 2004;31(6):734-43.

22. Bonner JA, Harari PM, Giralt J, Azarnia N, Shin DM, Cohen RB, et al. Radiotherapy plus cetuximab for squamous-cell carcinoma of the head and neck. The New England journal of medicine. 2006;354(6):567-78.

23. Brumund KT, Gutierrez-Fonseca R, Garcia D, Babin E, Hans S, Laccourreye O. Frontolateral vertical partial laryngectomy without tracheotomy for invasive squamous cell carcinoma of the true vocal cord: a 25-year experience. The Annals of otology, rhinology, and laryngology. 2005;114(4):314-22.

24. Dufour X, Hans S, De Mones E, Brasnu D, Menard M, Laccourreye O. Local control after supracricoid partial laryngectomy for "advanced" endolaryngeal squamous cell carcinoma classified as T3. Archives of otolaryngology--head \& neck surgery. 2004;130(9):1092-9.

25. Tufano RP. Organ preservation surgery for laryngeal cancer. Otolaryngologic clinics of North America. 2002;35(5):1067-80

26. Sheen TS, Ko JY, Chang YL. Partial vertical laryngectomy in the treatment of early glottic cancer. The Annals of otology, rhinology, and laryngology. 1998;107(7):593-7.

27. Yeager LB, Grillone GA. Organ preservation surgery for intermediate size (T2 and T3) laryngeal cancer. Otolaryngologic clinics of North America. 2005;38(1):11-20, vii.

28. Wein RO, Weber RS. The current role of vertical partial laryngectomy and open supraglottic laryngectomy. Current problems in cancer. 2005;29(4):201-14.

29. Steiner W, Ambrosch P, Hess CF, Kron M. Organ preservation by transoral laser microsurgery in piriform sinus carcinoma. Otolaryngology--head and neck surgery: official journal of American Academy of Otolaryngology-Head and Neck Surgery. 2001;124(1):58-67.

30. Ambrosch P, Kron M, Steiner W. Carbon dioxide laser microsurgery for early supraglottic carcinoma. The Annals of otology, rhinology, and laryngology. 1998;107(8):680-8.
31. Steiner W, Fierek O, Ambrosch P, Hommerich CP, Kron M. Transoral laser microsurgery for squamous cell carcinoma of the base of the tongue. Archives of otolaryngology--head \& neck surgery. 2003;129(1):36-43.

32. Ambrosch $\mathrm{P}$. The role of laser microsurgery in the treatment of laryngeal cancer. Current opinion in otolaryngology \& head and neck surgery. 2007;15(2):82-8.

33. O'Malley BW, Jr., Weinstein GS, Snyder W, Hockstein NG. Transoral robotic surgery (TORS) for base of tongue neoplasms. The Laryngoscope. 2006;116(8):1465-72.

34. Silver CE, Beitler JJ, Shaha AR, Rinaldo A, Ferlito A. Current trends in initial management of laryngeal cancer: the declining use of open surgery. European archives of oto-rhino-laryngology: official journal of the European Federation of Oto-Rhino-Laryngological Societies. 2009;266(9):1333-52.

35. Cooper JS, Porter K, Mallin K, Hoffman HT, Weber RS, Ang KK, et al. National Cancer Database report on cancer of the head and neck: 10-year update. Head \& neck. 2009;31(6):748-58.

36. Chen AY, Schrag N, Hao Y, Stewart A, Ward E. Changes in treatment of advanced oropharyngeal cancer, 1985-2001. The Laryngoscope. 2007;117(1):16-21.

37. Hoffman HT, Porter K, Karnell LH, Cooper JS, Weber RS, Langer CJ, et al. Laryngeal cancer in the United States: changes in demographics, patterns of care, and survival. The Laryngoscope. 2006;116(9 Pt 2 Suppl 111):1-13.

38. Chen AY, Halpern M. Factors predictive of survival in advanced laryngeal cancer. Archives of otolaryngology--head \& neck surgery. 2007;133(12):1270-6.

39. American Society of Clinical O, Pfister DG, Laurie SA, Weinstein GS, Mendenhall WM, Adelstein DJ, et al. American Society of Clinical Oncology clinical practice guideline for the use of larynx-preservation strategies in the treatment of laryngeal cancer. Journal of clinical oncology: official journal of the American Society of Clinical Oncology. 2006;24(22):3693-704

40. Beitler JJ, Cooper JS. Seduction by induction? Journal of clinical oncology: official journal of the American Society of Clinical Oncology. 2009;27(1):9-10. 